\title{
Prospects for Future Exploration of the Trans-Neptunian Region
}
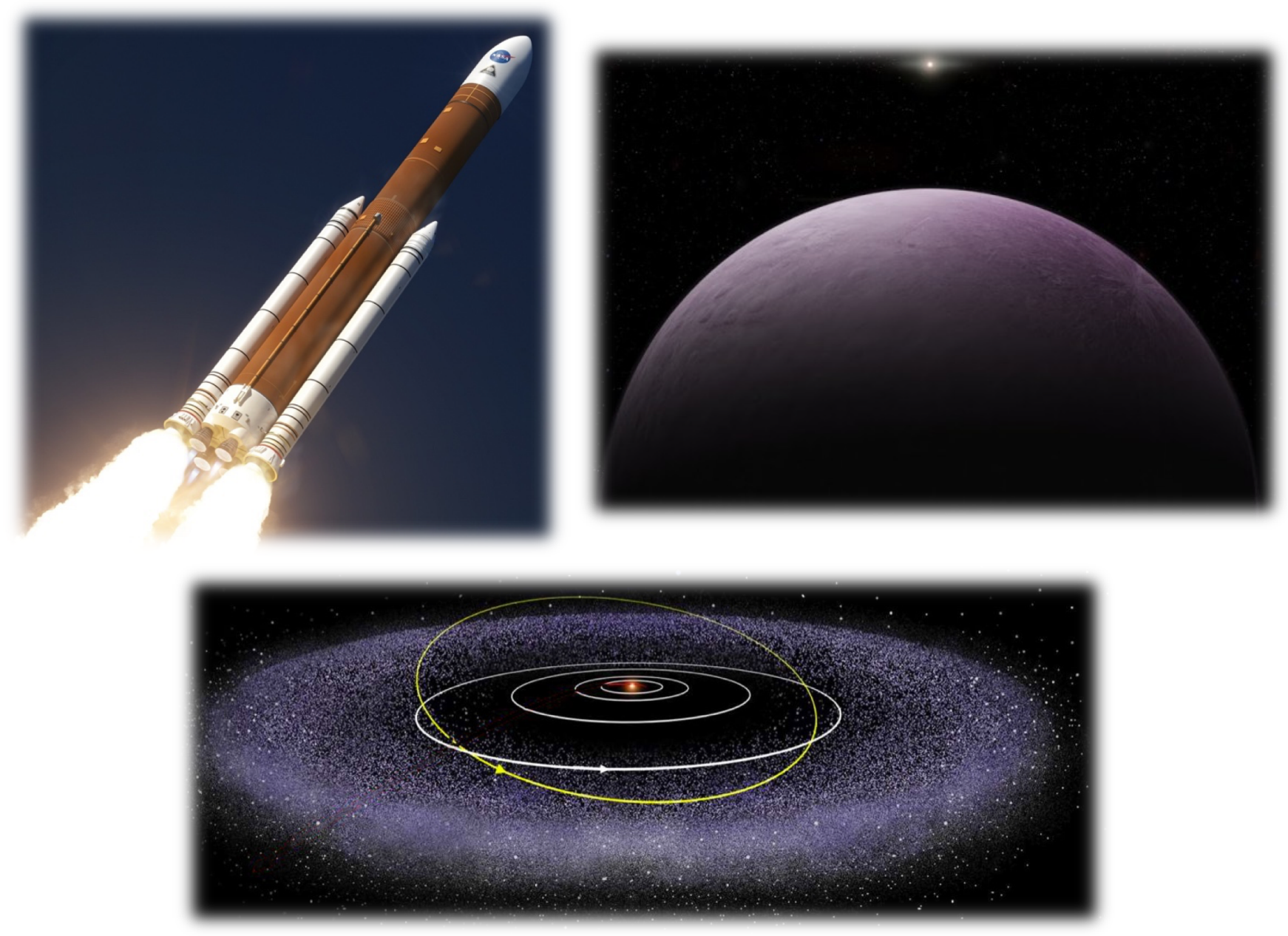

Primary Authors: Bryan J. Holler (STScI), Michele T. Bannister (U. Canterbury), Kelsi N. Singer (SwRI), S. Alan Stern (SwRI)

Co-Authors: Susan D. Benecchi (PSI), Cristina M. Dalle Ore (NASA Ames), Leigh N. Fletcher (U. of Leicester), Aurélie Guilbert-Lepoutre (U. de Lyon), Csaba Kiss (Konkoly Obs.), Pedro Lacerda (Queen's U. Belfast), Kathleen E. Mandt (JHU/APL), Michaël Marsset (MIT), Alex H. Parker (SwRI), Noemí Pinilla-Alonso (FSI/UCF), Darin Ragozzine (BYU), Mark B. Tapley (SwRI), Chadwick A. Trujillo (NAU), Orkan M. Umurhan (SETI, NASA Ames), Hajime Yano (JAXA ISAS), Leslie A. Young (SwRI)

Co-Signers: Richard Cartwright (SETI), Dale P. Cruikshank (NASA Ames), Estela Fernández-Valenzuela (FSI/UCF), Candy Hansen (PSI), Dean Hines (STScI), Jason D. Hofgartner (NASA/JPL), Timothy Holt (U. Southern Queensland), Carly Howett (SwRI), Katherine Murray (STScI), Cathy B. Olkin (SwRI), Nuno Peixinho (U. Coimbra), Alena Probst (JPL), Kirby D. Runyon (JHU/APL), Scott S. Sheppard (Carnegie Inst.), Anne Verbiscer (UVA), Maya D. Yanez (USC) 
Executive Summary: A strong case can be made for a flyby mission through the outer solar system in the coming decades. The census of the larger worlds of the trans-Neptunian region has shown that they display incredible diversity. Surface geology and composition, shapes, and binarity are far more varied in these distant populations than in many other regions of the solar system. Fully understanding these keystone worlds requires in situ observation. As demonstrated by New Horizons, the first trans-Neptunian flyby mission, close-up investigation can determine surface geology, atmospheric processes, space weather environment, interior-surface and surfaceatmosphere interactions, and provide constraints on a body's interior [1-3]. The narrative of the trans-Neptunian region is far from complete, and a deeper understanding requires visiting additional worlds in this region with spacecraft. Many of the known dwarf planets, which present a wide range of physical and compositional diversity, are presently at mission-accessible heliocentric distances of $\mathrm{r}<100 \mathrm{au}$. The sky has been thoroughly mapped for bright $\mathrm{r}<100$ au transNeptunian objects (TNOs) outside of the galactic plane [4]. Upcoming surveys including the Vera C. Rubin Observatory's Legacy Survey of Space and Time (LSST) [5] will complete this effort for fainter, smaller objects (see white paper by Jones et al.), and also provide a more complete picture of the populations of minor bodies that could be flyby targets en-route to larger worlds. Of the $6 \times 10^{6}$ minor planets soon to be cataloged, some 40,000 of them lie beyond Neptune [6]. Thus, future missions must be conceived as ensemble routes that encounter a diverse collection of targets.

To more fully understand the bounty of trans-Neptunian worlds, exploration missions of the 2020s and beyond will all be grand tours, visiting a variety of objects.

Synergies with Ice Giant and Centaur exploration: The 2013-2022 Planetary Science Decadal Survey included an ice giant mission as the next priority Flagship after a Mars Sample Return lander and a reduced Europa mission. For practical trajectory reasons, a Uranus Orbiter with Probe was recommended, but a mission for either ice giant was desired. In response, the US science community held a workshop in 2014 to develop a suite of science objectives, encompassing the full exploration of the ice giant systems, including the atmospheres, magnetospheres, rings, and satellites. While similar objectives were found for both Uranus and Neptune, Triton was specifically called out for further study due to its geologic activity (see white paper by Hansen et al.). As a probable captured TNO dwarf planet [7], Triton's bulk composition and origin are akin to other TNOs. Triton then experienced a radically different evolution, so visiting Triton achieves different, complementary goals from visiting a TNO still residing in the Kuiper Belt.

Exploration of the cold, icy worlds in the distant solar system cannot be considered in isolation, and synergies exist between TNO exploration and the efforts to send robotic missions to the two ice giants, Uranus and Neptune.

One possible approach for missions to Uranus and Neptune would be through combined outer solar system missions. A Neptune orbiter with multiple Triton flybys could include a Centaur flyby en-route, for comparison with Trojans and TNOs. In the same manner, a mission to the transNeptunian region could include a Uranus flyby and gravity assist. These two missions will have then explored multiple populations of outer solar system small bodies. These options are further discussed in Simon et al., 2018. Coordination with missions focused on heliophysics and astrophysics goals is also promising [8-10] (and the white paper by Runyon et al.).

Centaurs are recent escapees from the Kuiper Belt [11-13], with semi-major axes between the giant planets (5.5-30.1 au). A single flyby reconnaissance mission to multiple Centaurs can significantly multiply the number and diversity of TNOs studied in detail beyond the single TNO, 
Arrokoth, that New Horizons explored in 2019 [3,14]. Such a mission can study the geology and other surface properties of a variety of bodies from the Kuiper Belt with different sizes, colors, compositions, and densities. Because many Centaurs are 3-5 times closer to Earth than TNOs, they can be more quickly reached for flybys or more detailed orbital reconnaissance missions than direct missions to the Kuiper Belt. Three potential mission architectures for Centaur exploration are:

- A dedicated Centaur tour reaching multiple Centaurs with a single spacecraft. The Centaurus mission [15] was proposed to the most recent NASA Discovery call with a proposed launch in 2028, a flyby of Schwassmann-Wachmann 1 in 2032, and Chiron in 2045. This mission design is representative of a broad range of possible Centaur reconnaissance missions, with the distinct possibility of flying by multiple Centaurs. Given a velocity of $\sim 3 \mathrm{au} / \mathrm{yr}$ and a launch in 2030 , any Centaur within $\sim 11$ au could reasonably be reached using solar power.

- A serendipitous Centaur flyby on the way to an ice giant or trans-Neptunian dwarf planet. Although possible in principle (Table 1), we are not aware of specific mission studies that demonstrate a viable Centaur en-route to an ice giant or trans-Neptunian dwarf planet.

- A Centaur orbiter, with additional Centaur flybys en-route. A Chiron orbiter was studied for the 2013-2022 Decadal Survey [16].

We recommend that further studies be undertaken to consider recent developments such as the Space Launch System (SLS), new and emerging propulsion technologies, current- and nextgeneration instrumentation, and an increasingly wide range of Centaur targets.

The exploration of Centaurs by spacecraft represents a powerful way to study TNOs because Centaurs are more accessible than bodies in the Kuiper Belt itself. The exploration of Centaurs can advance many important aspects of Kuiper Belt and TNO science.

TNO dwarf planets: The dwarf planets Pluto, Eris, Makemake, and Haumea are the largest currently known TNOs. Each dwarf planet presents the unique opportunity to study a wide variety of important processes at large heliocentric distances, including:

Physical and chemical processes such as volatile transport, albedo feedback, and creation of complex organics on distant surfaces. High spectral resolution, high-SNR observations have revealed bands of irradiation products overlain on top of Makemake's clear $\mathrm{CH}_{4}$ signature: $\mathrm{C}_{2} \mathrm{H}_{6}$ (ethane), $\mathrm{C}_{2} \mathrm{H}_{4}$ (ethylene), $\mathrm{C}_{2} \mathrm{H}_{2}$ (acetylene), and possibly $\mathrm{C}_{3} \mathrm{H}_{8}$ (propane) and higher mass alkanes [17]. These are tracers of the irradiation process that transforms $\mathrm{CH}_{4}$ into longer-chain hydrocarbons and then into the red, dark macromolecular, carbonaceous materials often identified as refractory organic material [18]. In light of this, Makemake's overall exceptionally high albedo, $\sim 0.77$ [19], is puzzling and has been hypothesized as due to a resurfacing mechanism, which could be similar to the one acting in Sputnik Planitia on Pluto, but on a much larger scale [20]. A close look at its albedo distribution and color would reveal the true distribution of dark and bright material, providing clues on past ice migration patterns, and enabling a direct comparison to Pluto.

Atmospheric processes such as winds, production of haze, atmospheric escape rates, and collapse. In contrast to Eris' high albedo, its satellite Dysnomia has an albedo of only 4\% [21]. This large difference between Eris and Dysnomia is not surprising, given that Eris' surface is dominated by volatile ices possibly refreshed by volatile transport in the present-day [22]. What is surprising is the extremely low albedo of Dysnomia, below the TNO average of 8\% [23]. [24] describe a process by which Charon's poles redden and darken by collection and radiolysis of $\mathrm{CH}_{4}$ escaping from Pluto's atmosphere. Eris represents a similar reservoir of $\mathrm{CH}_{4}$ and at the colder temperatures experienced by this system, volatile $\mathrm{CH}_{4}$ could be more easily retained on Dysnomia, 
and not just at polar cold traps. Visible and infrared full-surface imaging and spectroscopy of Dysnomia could result in further exploration of this mechanism at another dwarf planet.

Geologic processes and features like cratering, tectonics, cryovolcanism, plumes, and subsurface oceans. Haumea has a surface of pure water ice [25-29] and the shortest known rotation period $(\mathrm{P}=3.9154 \pm 0.0002 \mathrm{hr})$ [30] of the $>100 \mathrm{~km}$-sized objects in the solar system, which has formed it into a highly elongated triaxial ellipsoid [30-32]. As the only large body spinning close to "breakup," a variety of questions can be investigated via the highly detailed shape, topography, and gravity field information that can only be measured by a mission, including: (1) the behavior of icy material under unusual stress fields and what that implies for a body's shape, (2) the characteristics of large scale topography in the low-gravity equatorial regions, and (3) the shapes of differentiated layers in the case of extreme rotation. Much about Haumea's collision would be learned by the shape of its craters as a function of size and location, the presence and direction of faults, and other topographic and gravimetric information. Haumea is the largest member of the only known trans-Neptunian collisional family [33] and supports a ring system [32].

Satellite formation and evolution through comparison with the primary, tidal evolution, and non-gravitational primary-secondary interactions. Of the 10 largest known TNOs, 9 are known to have at least one satellite. Additionally, these systems are all very similar: small satellites orbiting relatively close to their primaries. This is in contrast to the smallest known binary TNOs, which almost all have comparably sized components and large relative separations (Fig. 1). This suggests different formation mechanisms between large and small TNO binary systems [21,34-40]. In situ study of both types of binaries are necessary for understanding the formation circumstances and collisional environment of the early trans-Neptunian region; this could potentially be achieved on a trajectory for flyby of both a dwarf planet and at least one Arrokoth-like cold classical KBO.

Each TNO dwarf planet presents the unique opportunity to study a wide variety of important physical, chemical, and geological processes at large heliocentric distances.

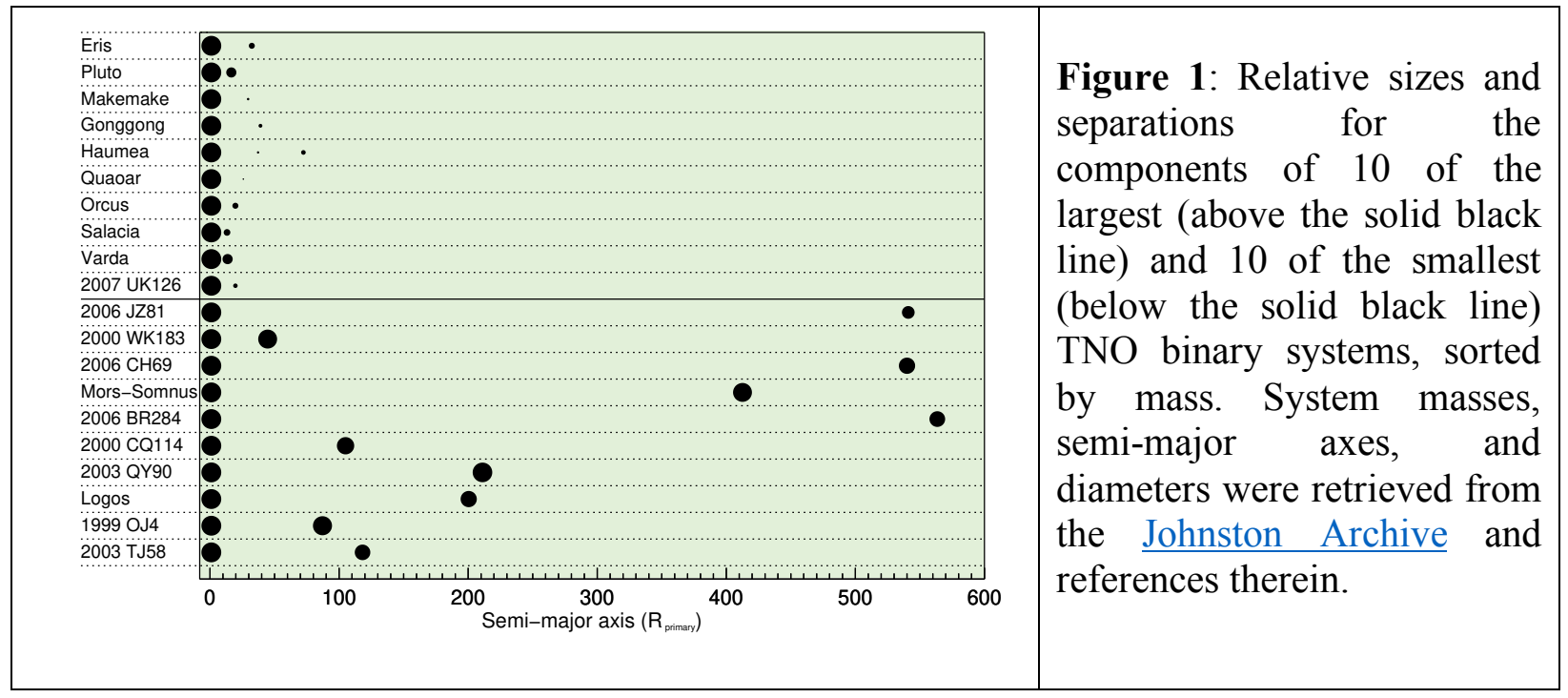

Mid-sized and small TNOs: Mid-sized TNOs lie in the diameter range between the geologically active dwarf planets and the collisionally evolved small bodies $(500 \mathrm{~km} \lesssim \mathrm{D} \lesssim 1200 \mathrm{~km})$. A flyby of one or more of these TNOs, or Centaurs like Chiron and Chariklo (TNOs at closer heliocentric distances), would provide key constraints on the original protoplanetary disk and the mechanisms involved in their formation. We highlight some mid-sized TNOs of particular interest below. 
Sedna is the largest known member of the population of high-perihelia (q>50 au) "extreme" TNOs [41-44]. With a perihelion distance of 76 au and an aphelion of $931 \mathrm{au}$, Sedna spends most of its time outside the heliosphere and is frequently referred to as an inner Oort cloud object; however, it is approaching perihelion in 2076 and is currently only 84 au from the Sun. Sedna is also extremely red, a TNO property that is typically associated with refractory organic materials. Sedna thus represents a unique laboratory for studying the effects of the interstellar radiation environment on the surface properties and compositions of TNOs.

Icy objects showing evidence for geological activity are generally the subject of intense investigation, since it hints at the persistence of fluid either in subsurface oceans or as localized pockets of fluid in an icy mantle. There is reason to believe that the surfaces of some TNOs may show geologic activity, giving a window into the past and present. Such features require high spatial resolution imaging: they are typically not observable from Earth-based facilities. Subsurface oceans are of extreme interest from an astrobiological context and could be present on the dwarf-planet class objects Triton, Pluto, and Charon [45-49]. For the mid-sized TNOs, Orcus, is a prime target for past or current geologic activity, specifically cryovolcanic activity. Cryovolcanism could be driven by an ammonia-water mixture that could melt at temperatures as low as $175 \mathrm{~K}$, which are achievable in the interiors of many dwarf planet-class TNOs [50]. Orcus is smaller than Charon $(\sim 920 \mathrm{~km}$ vs. $\sim 1200 \mathrm{~km})[1,51]$, but both objects' surfaces are dominated by water ice, with the presence of other species reported, such as $\mathrm{NH}_{3}$ (ammonia), $\mathrm{NH}_{3} \cdot \mathrm{nH}_{2} \mathrm{O}$ (ammonia hydrates), and perhaps $\mathrm{NH}^{+}{ }_{4}$ (ammonium) and $\mathrm{C}_{2} \mathrm{H}_{6}$ (ethane) [52-55]. The density of Orcus is lower than that of Charon $\left(\sim 1.7 \mathrm{~g} \mathrm{~cm}^{-3}\right)$ [1], yet models highlight the possibility for subsurface oceans to be formed in both $[48,49,53,56,57]$.

\section{Mid-sized TNOs are the last category of outer solar system objects yet to be visited by a spacecraft. Their physical properties - including surface composition, crater populations, and bulk density - may hold clues to many mysteries of early solar system evolution.}

One TNO that defies categorization is Lempo (1999 $\left.\mathrm{TC}_{36}\right)$. While both resolved and contact binaries are common in the Kuiper belt, the Lempo system is the only TNO hierarchical triple with an inner tight, equal-mass binary (two $\sim 200 \mathrm{~km}$ objects, Lempo and Hiisi, orbiting $\sim 800$ $\mathrm{km}$ apart) orbited by a $\sim 100 \mathrm{~km}$ object, Paha, $\sim 7000 \mathrm{~km}$ away [58,59]. The origin of this configuration is unclear. If the Lempo/Hiisi binary is a failed contact binary, the role of Paha is important to understand. Two mechanisms have been suggested for the origin of Paha: gravitational capture and collision [58]. Capture of Paha into its current co-planar orbit is unlikely, but the angular momentum of the Lempo system is higher than that of the other known TNO multiple systems, Pluto and Haumea, that likely formed by impact. Contact binaries such as Arrokoth likely formed by very slow speed collisions between components $(\sim 2 \mathrm{~m} / \mathrm{s}$, comparable to human walking speed) [14]. Could this also lead to the contact binary being susceptible to fragmentation following a giant impact or gravitational capture of Paha? In situ surface mapping of Lempo and Hiisi would provide geomorphological clues for a more complete evaluation.

The abundant small TNOs on low-inclination orbits offer an excellent opportunity to explore the primordial distribution of planetesimal sizes, directly probing the least-altered products of the ancient gas and dust disk from the dawn of the solar system.

Due to a break from a very steep to a very shallow size distribution, objects near the break size $(\mathrm{D} \sim 100 \mathrm{~km})$ represent the peak in the mass distribution of the trans-Neptunian region. By comparison to observations of other current and past TNOs (including Centaurs and captured 
irregular satellites), we can establish which extant properties of outer solar system small bodies are most shaped by their origins versus their environment and history. Studying the properties of small TNOs and contrasting them against the properties of larger TNOs and similarly-sized objects in less pristine populations, also provides a means of determining the extent of collisional processing within this population. The flyby of Arrokoth [60-62] shows that parts of the Kuiper Belt have experienced remarkably limited collisional processing. These small worlds appear to represent the primordial distribution of planetesimal sizes. The smaller the threshold at which TNOs transition from dominantly primordial objects to collisional fragments, the greater the number of cosmogonically-relevant targets that are available for the multi-flyby missions that will become the dominant modality of design after LSST's mapping in the first 3 years of that survey.

The flyby of the small (18-km equivalent spherical diameter) cold classical KBO (CCKBO) $2014 \mathrm{MU}_{69}$ (Arrokoth) by New Horizons revealed landmark results regarding both small KBO geology and composition and planetesimal accretion [14]. Among the most important results obtained was the convincing case $[14,63]$ for its formation by pebble cloud collapse/streaming instabilities. The implications of this finding are deep, but cannot be confidently assumed for all Kuiper Belt planetesimals until a wider suite of such bodies has been explored. Since such bodies are numerous, Monte Carlo studies have revealed that future missions to Pluto, other dwarf planets, or even mid-sized TNOs can expect to easily reach one or even several "MU ${ }_{69}$ s." Such flybys will also allow better separation of the trans-Neptunian impactor flux by sub-population (Plutinos, CCKBOs, scattered disk, etc.) than New Horizons could provide with a single TNO flyby from a single sub-population.

Table 1: Example mission targets and encounter dates

\begin{tabular}{|c|c|c|}
\hline Flyby dates & $\begin{array}{l}\text { Gas Giant } \\
\text { Primary TNO target } \\
\text { [secondary TNO] }\end{array}$ & Comments \\
\hline 2045-06-05 & Uranus & \\
\hline $2049-07-23$ & $2003 \mathrm{AZ}_{84}$ & No secondary targets; $3: 2$ resonant \\
\hline 2051-05-15 & Varuna & Hot classical KBO \\
\hline $2042-10-02$ & {$\left[2014 \mathrm{SB}_{349}\right]$} & Centaur \\
\hline $2059-09-08$ & $2002 \mathrm{UX}_{25}$ & Hot classical KBO \\
\hline $2043-05-07$ & {$\left[2004 \mathrm{PA}_{44}\right]$} & Centaur \\
\hline $2048-04-25$ & [2015 RU 245$]$ & Centaur \\
\hline 2049-04-13 & [2012 UV 177$]$ & Neptune Trojan (L4) \\
\hline 2053-04-08 & {$\left[2014 \mathrm{TM}_{95}\right]$} & $4: 3$ resonant \\
\hline 2040-04-05 & Neptune & \\
\hline $2046-05-16$ & $2015 \mathrm{RR}_{245}$ & $9: 2$ resonant \\
\hline $2043-08-14$ & {$\left[2003 \mathrm{QS}_{91}\right]$} & Cold classical KBO \\
\hline $2046-12-19$ & Salacia & No secondary targets; hot classical KBO \\
\hline 2047-06-09 & Teharonhiawako & Cold classical KBO \\
\hline $2044-07-27$ & {$\left[2002 \mathrm{PU}_{170}\right]$} & $2: 1$ resonant \\
\hline $2060-03-06$ & Eris & Scattered Disk Object (SDO) \\
\hline $2043-08-12$ & [2003 QS91] & Possible secondary for $2015 \mathrm{R}_{245}$ flyby too \\
\hline 2043-10-18 & {$\left[2014 \mathrm{OC}_{394}\right]$} & Alternate to 2003 QS91 \\
\hline $2044-02-27$ & {$\left[2005 \mathrm{PF}_{23}\right]$} & Cold classical KBO \\
\hline 2044-03-06 & [2004 PE 112$]$ & Cold classical KBO \\
\hline 2044-03-30 & {$\left[2005 \mathrm{PH}_{23}\right]$} & Cold classical KBO \\
\hline 2044-06-01 & {$\left[2005 \mathrm{PO}_{21}\right]$} & Cold classical KBO \\
\hline 2044-09-07 & [2003 QT90] & Cold classical KBO \\
\hline 2044-10-05 & [2006 UM $\left.\mathrm{UM}_{321}\right]$ & Cold classical KBO \\
\hline 2045-04-13 & {$\left[2000 \mathrm{PS}_{30}\right]$} & Scattered Disk Object (SDO) \\
\hline 2046-02-07 & [2013 VZ31] & Cold classical KBO \\
\hline
\end{tabular}


Multi-target flyby trajectories: A flyby of an ice giant can capitalize on a gravity assist to redirect to a TNO or Centaur. If a modest propulsion system is added to the vehicle, secondary targets may also become available, further multiplying the mission's effectiveness. By selecting a reasonable set of constraints and then showing a method to construct several example multi-target trajectories, the feasibility of this class of mission can be demonstrated. (Other mission options can be seen in [64].) We evaluated high-value TNO targets (Table 1) accessible within 20 years of a giant planet flyby assuming a Jupiter gravity assist to Uranus or Neptune with a ballistic trajectory post-Jupiter flyby, a launch characteristic energy (C3) $<100 \mathrm{~km}^{2} / \mathrm{s}^{2}$, and a 30 -year mission lifetime. We assume launch around 2030, with planning and development of the mission in the 2020s.

Pioneers 10 and 11 and both Voyagers exceeded their 20-year mission lifetimes following their final planetary flyby, and like the missions we investigated, took $\sim 10$ years to accomplish their giant planet flybys. Other spacecraft also routinely exceed this duration.

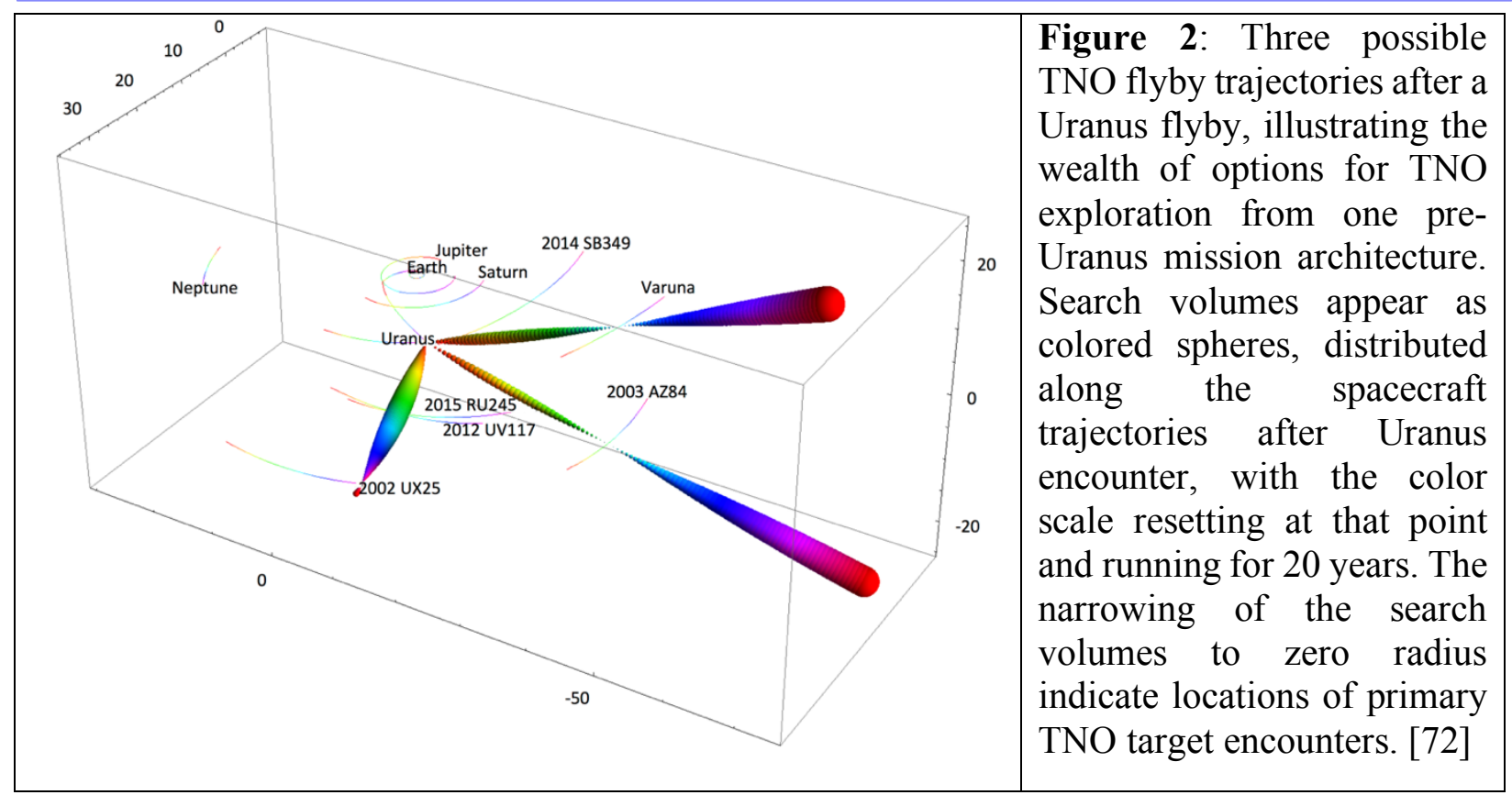

We concentrated on locating credible multi-target missions with reasonable engineering parameters and located an array of potential missions. For all cases, we verified that the giant planet flyby had a periapse greater than the planetary radius. Three primary targets of interest are accessible after a Uranus flyby (2003 AZ 84 , Varuna, $2002 \mathrm{UX}_{25}$; Fig. 2), and four are accessible after a Neptune flyby (2015 RR 245 , Salacia, Teharonhiawako, Eris). Most trajectories have "secondary" targets available prior to close encounters with the primary targets. Table 1 summarizes dates and targets for the trajectories, including potential secondaries. Eris is available after a Neptune gravitational assist, though it requires all but one month of the arbitrary 20-year deadline to reach. A combined mission to $2015 \mathrm{RR}_{245}$, a mid-sized TNO ( 670 km) [65-67], and Eris is only barely outside the selected parameters. Note that the trajectories from Uranus to 2002 $\mathrm{UX}_{25}$, and from Neptune to Eris, have two possible secondaries each. While it is unlikely that a single mission could fly by both targets, long-range imaging would be able to study the target not selected for an encounter in greater detail than potential Earth-based observations, as demonstrated by New Horizons [68-71]. The Rubin Observatory's LSST will increase the en-route flyby opportunities by an order of magnitude [6]. That we have so many potential choices at present reinforces that available trajectories will multiply beyond current conception in the mid-2020s. 
The Rubin Observatory's LSST will provide an incredible order of magnitude increase in the known populations in the outer solar system - early in its survey. We ask that the Decadal recommend investment to support the development of multi-flyby missions to this incoming treasure trove of new, scientifically key destinations.

References: [1] Stern, S.A., et al. 2015. Science 350, aad1815. [2] Stern, S.A., et al. 2018. ARAA 56, 357. [3] Stern, S.A., et al. 2018. SSRv 214, 77. [4] Holman, M.J., et al. 2018. ApJ 855, L6. [5] Ivezić, Z., et al. 2018. arXiv:0805.2366. [6] Schwamb, M.E., et al. 2018. arXiv:1802.01783. [7] Agnor, C.B., Hamilton, D.P. 2006. Nature, 441, 192. [8] Mandt, K., et al. 2018. AGU, Vol. 2018, SH33C-3666. [9] Mandt, K.E., et al. 2019. LPSC, Vol. 50, 2709. [10] Zemcov, M., et al. 2018. PASP 130, 115001. [11] Fernandez, J.A. 1980. MNRAS 192, 481. [12] Levison, H.F., Duncan, M.J. 1997. Icarus 127, 13. [13] Sarid, G., Prialnik, D. 2009. M\&PS 44, 1905. [14] Stern, S.A., et al. 2019. Science 364, aaw9771. [15] Singer, K.N., et al. 2019. EPSC-DPS2019-2025. [16] Adams, M.L., et al. 2010. 2013-2022 PDS. [17] Brown, M.E., et al. 2015. AJ 149, 105. [18] Cruikshank, D.P., et al. 2005. ASR 36, 178. [19] Ortiz, J.L., et al. 2012. Nature 491, 566. [20] Grundy, W.M., Umurhan, O.M. 2017. DPS 49, \#202.02. [21] Brown, M.E., Butler, B.J. 2018. AJ 156, 164. [22] Hofgartner, J.D., et al. 2019. Icarus 334, 52-61. [23] Stansberry, J.A., et al. 2012. Icarus 219, 676. [24] Grundy, W.M., et al. 2016. Nature 539, 65. [25] Licandro, J., et al. 2006. A\&A 457, 329. [26] Trujillo, C.A., et al. 2007. ApJ 655, 1172. [27] Pinilla-Alonso, N., et al. 2007. A\&A 468, L25. [28] Pinilla-Alonso, N., et al. 2008. A\&A 489, 455. [29] Pinilla-Alonso, N., et al. 2009. A\&A 496, 547. [30] Rabinowitz, D.L., et al. 2006. ApJ 639, 1238. [31] Lockwood, A.C., et al. 2014. EM\&P 111, 127. [32] Ortiz, J.L., et al. 2017. Nature 550, 219. [33] Brown, M.E., et al. 2007. Nature 446, 294296. [34] Weidenschilling, S.J. 2002. Icarus 160, 212. [35] Goldreich, P., et al. 2002. Nature 420, 643. [36] Funato, Y., et al. 2004. Nature 427, 518. [37] Astakhov, S.A., et al. 2005. MNRAS 360, 401. [38] Canup, R.M. 2005. Science 307, 546. [39] Nesvorný, D., et al. 2010. AJ 140, 785. [40] Nesvorný, D., et al. 2019. Nat. Ast. 3, 808. [41] Brown, M.E., et al. 2004. ApJ 617, 645. [42] Trujillo, C.A., Sheppard, S.S. 2014. Nature 507, 471. [43] Bannister, M.T., et al. 2017. AJ 153, 262. [44] Sheppard, S.S., et al. 2019. AJ 157, 139. [45] Gaeman, J. et al. 2012. Icarus 220, 339347. [46] Robuchon, G., Nimmo, F. 2011. Icarus 216, 426-439. [47] Kimura, J., Kamata, S. 2020. P\&SS 181, 104828. [48] Malamud, U., Prialnik, D. 2015. Icarus 246, 21. [49] Desch, S.J., Neveu, M. 2017. Icarus 287, 175. [50] Stevenson, D.J. 2004. Nature 432, 681. [51] Fornasier, S., et al. 2013. A\&A 555, A15. [52] Barucci, M.A., et al. 2008. A\&A 479, L13. [53] Delsanti, A., et al. 2010. A\&A 520, A40. [54] DeMeo, F.E., et al. 2010. A\&A 521, A35. [55] Carry, B., et al. 2011. A\&A 534, A115. [56] Shchuko, O.B., et al. 2014. P\&SS 104, 147. [57] Desch, S.J. 2015. Icarus 246, 37. [58] Benecchi, S.D., et al. 2010. Icarus 207, 978. [59] Mommert, M., et al. 2012. A\&A 541, A93. [60] Nesvorný, D., et al. 2011. AJ 141, 159. [61] Parker, A.H., Kavelaars, J.J. 2012. ApJ 744, 139. [62] Singer, K.N., et al. 2019. Science 363, 955. [63] McKinnon, W.B., et al. 2020. Science 367, aay6620. [64] Zangari, A.M., et al. 2019. JSR 56, 919. [65] Bannister, M.T., et al. 2016. AJ 152, 212. [66] Bannister, M.T., et al. 2016. AJ 152, 70. [67] Bannister, M.T., et al. 2018. ApJ 236, 18. [68] Porter, S.B., et al. 2016 ApJL 828, L15. [69] Porter, S.B., et al. 2018. DPS 50, \#509.07. [70] Verbiscer, A., et al. 2018. DPS 50, \#509.08. [71] Weaver, H., et al. 2018. DPS 50, \#311.03. [72] Bannister, M.T., et al., submitted to SSRv. 